\title{
一类算子方程解的存在及唯一性 ${ }^{*}$
}

\author{
李 寿 佛 \\ (湘洒大学数学系, 湘涪 411105)
}

\section{关组诃非线性算子方程、初值问题、多值多导法}

设 $X$ 是 Hilbert 空间, 其基域为 $K$ (实或复),$\langle\cdot, \cdot\rangle\rangle$ 是其中内积, $\|\cdot\|$ 是相应范数. 对任给 矩阵 $A-\left[a_{i i}\right] \in K^{p \times q}$, 相应可定义一线性映射 $\tilde{A}: X^{q} \rightarrow X^{p}$ :

$$
\tilde{A}_{u}-v-\left(\sum_{i=1}^{\dot{1}} a_{1 i} u_{i}, \sum_{j=1}^{q} a_{2 j} u_{i}, \cdots, \sum_{j=1}^{t} a_{p i} u_{i}\right) \in X^{p}, \forall u-\left(u_{1}, u_{2}, \cdots, u_{q}\right) \in X^{q} .
$$

设已给 $\sigma \in X^{\prime}, A_{i} \in K^{\prime \times s}$ 及连续映射 $\varphi_{j}: X^{\prime} \rightarrow X^{\prime}(j-1,2, \cdots, m)$, 考虑空间 $X^{\prime}$ 中 的算子方程

$$
\phi(x):=x-\sigma-\sum_{j=1}^{m} \tilde{A}_{i} \varphi_{i}(x)-0,
$$

这里 $x \in X^{s}$ 是末知元, 并设诸 $\varphi_{i}$ 满足

$$
\operatorname{Re}\left\langle\widetilde{D}_{i}\left(\varphi_{i}(y)-\varphi_{i}(z)\right), y-z\right\rangle \leqslant\left\langle y-z, X_{i}(y-z)\right\rangle, \forall y, z \in X^{c}, j-1,2, \cdots, m, \text { (2) }
$$

其中 $D_{j} 、 \Lambda_{j} \in K^{i \times s}$, 满足 $\Lambda_{j}^{*}-\Lambda_{j}$, 称它们为映射 $\varphi_{j}$ 的示性矩阵; $X^{s}$ 中的内积及范数定义为

$$
\langle y, z\rangle-\sum_{i=1}^{\dot{S}}\left\langle y_{i}, z_{i}\right\rangle,\|y\|-\left(\sum_{i=1}^{\prime}\left\|y_{i}\right\|^{2}\right)^{\dagger}, \forall y-\left(y_{1}, y_{2}, \cdots, y_{i}\right), z-\left(z_{1}, z_{2}, \cdots, z_{i}\right) \in X^{i} .
$$

引理 $1^{\text {tu }}$ 设 $\psi: X \rightarrow X$ 是连续映射, 满足

$$
\operatorname{Re}\langle\psi(u)-\psi(v), u-v\rangle \geqslant 0, \forall u, v \in X ; \operatorname{Re}\langle\psi(u), u\rangle \geqslant 0, \forall u \in X \backslash \bar{S}(0, \varepsilon) .
$$

则方程 $\psi(x)-0$ 存在一解 $x \in \bar{S}(0, \varepsilon):-\{x \in X:\|x\| \leqslant \varepsilon\}$ (这里设 $\varepsilon \geqslant 0$ ).

定理 1 若存在 Hermite 矩阵 $R \in K^{\prime \times s}$, 使下列条件至少有一个成立:

(i) $Q_{0}>0, Q_{1} \geqslant 0 ; \quad$ (ii) $Q_{0} \geqslant 0, Q_{1}>0$;

(iii) $Q_{0} \geqslant 0, Q_{1} \geqslant 0$, 且有一 $\varphi_{i}$, 使不等式(2)当 $y \neq z$ 时严格成立. 则方程(1)至多有一个解. 这里及下文中, 记

$$
\begin{gathered}
Q_{0}-R-\sum_{i=1}^{m} \Lambda_{i}, Q_{1}-\mathscr{D}^{*} \mathscr{A}+\mathscr{A}^{*} \mathscr{D}-2 \mathscr{A}^{*} R \mathscr{A}, \mathscr{D}-\left[D_{1}, D_{2}, \cdots, D_{m}\right], \\
\mathscr{A}-\left[A_{1}, A_{1}, \cdots, A_{m}\right],
\end{gathered}
$$

$\lambda_{\text {max }}^{\circ} \lambda_{\text {min }}^{2}$ 表示矩阵 $Q$ 的最大及最小特征值, $Q \geqslant 0$ (或 $>0$ ) 表示矩阵 $Q$ 非负定(或正定).

证 若方程 (1) 有二相异解 $y, z$, 记

$$
\nu-\left(\varphi_{1}(y)-\varphi_{1}(z), \varphi_{2}(y)-\varphi_{2}(z), \cdots, \varphi_{-}(y)-\varphi_{m}(z)\right) \in X^{\prime \prime},
$$

则当条件 (i)、(ii)、(iii) 中至少有一个成立时, 从(1)、(2)式可推出

本文1991年4月12日收到. 1991 年10月25 日收到修改稆.

- 园家自然科学基金资助项目. 


$$
\mathscr{A}:-\sum_{i=1}^{A} \operatorname{Re}\left\langle\check{D}_{i}\left(\varphi_{i}(y)-\varphi_{i}(z)\right), y-z\right\rangle-\frac{1}{2}\left\langle\nu,\left(\check{\mathscr{D}}^{*} \mathscr{A}+\mathscr{A} * \mathscr{\mathscr { D }}\right) v\right\rangle<0,
$$

但此式与下面等式相矛盾:

$$
\mathscr{A}-\operatorname{Re}\left\langle\tilde{\mathscr{D}}_{v}, y-z\right\rangle-\operatorname{Re}\left\langle\tilde{\mathscr{D}}_{\nu}, \mathscr{A} v\right\rangle-\operatorname{Re}\left\langle\mathscr{D}_{v}, \phi(y)-\phi(x)\right\rangle-0 .
$$

定理 2 若定理 1 中条件(i)或(ii)成立, 且集合 $M-\left\{p \in \mathrm{N}: p \leqslant m, \operatorname{Det}\left(A_{p} D_{p}\right) \neq\right.$ $0\}$ 非空, 则方程 (1)有唯一解 $\hat{x} \in X^{\prime}$, 且有估计

$$
\left\|\hat{x}-x_{0}\right\| \leqslant \lambda^{-1} \min _{p \in \mathcal{N}}\left\|\tilde{D}_{p} \tilde{A}_{p}^{-1} \phi\left(x_{0}\right)\right\|, \forall x_{0} \in X^{s} .
$$

这里

$$
\lambda-\lambda_{\text {mia }}^{q_{j}}+\lambda_{\text {mia }}^{Q_{i a}} / \lambda_{\max }^{2 x_{x}^{*}}>0 .
$$

证 任给 $p \in M, x_{0} \in X^{s}$. 令 $y-x-x_{0}$. 则(1) 式等价于

$$
\phi_{p, x_{0}}(y):-\widetilde{D}_{p} \tilde{A}_{p}^{-1} \phi\left(y+x_{0}\right)=0 \text {. }
$$

$\forall y, z \in X^{s}$, 令 $u-\left(u_{1}, u_{2}, \cdots, u_{m}\right) \in X^{\prime m}$, 其中

$$
u_{i}-\varphi_{j}\left(y+x_{0}\right)-\varphi_{i}\left(z+x_{0}\right) \text {, 当 } j \neq p ; u_{p}-\tilde{A}_{p}^{-1}\left(y-z-\sum_{\substack{i \neq 1 \\ i \neq \rho}}^{\infty} \tilde{A}_{i} v_{i}\right) \text {. }
$$

当定理 1 中条件(i)或(ii)成立时, 由(1)、(2)、(3)、(6)、(7)式得

$$
\begin{aligned}
& \operatorname{Re}\left\langle\phi_{p, x_{0}}(y)-\phi_{p, x_{j}}(z), y-z\right\rangle-\operatorname{Re}\langle\widetilde{\mathscr{D}} u, \tilde{\mathscr{A}} u\rangle-\operatorname{Re}\left\langle\sum _ { i = 1 } ^ { \infty } \widetilde { D } _ { i } \left(\varphi_{i}\left(y+x_{0}\right)\right.\right. \\
& \left.\left.-\varphi_{i}\left(z+x_{0}\right)\right), y-z\right\rangle \\
& \geqslant\langle u, \mathscr{A} * \tilde{R} \tilde{A} u\rangle+\frac{1}{2} \lambda_{\mathrm{min}}^{\rho_{\mathrm{in}}}\|u\|^{2}-\sum_{i=1}^{n}\left\langle y-z, \tilde{\Lambda}_{i}(y-z)\right\rangle \\
& \geqslant\left\langle y-z, \widetilde{Q}_{0}(y-z)\right\rangle+\lambda_{\operatorname{mia}}^{\rho_{1}}(2 \lambda \underset{\max }{*})^{-1}\|y-z\|^{2} \geqslant \lambda\|y-z\|^{2}
\end{aligned}
$$

因而

$$
\operatorname{Re}\left\langle\phi_{p, x_{0}}(y), y\right\rangle \geqslant 0, \forall y \in X^{s} \backslash \bar{S}\left(0,\left\|\phi_{p, x_{0}}(0)\right\| / \lambda\right) .
$$

由于 $\phi_{p, x_{0}}$ 连续, 从(5)、(8)、(9)式及引理 1 推知方程 (6)存在一解 $y_{p, x} \in \bar{S}\left(0, \lambda^{-1} \| \phi_{p, x_{0}}(0) i i\right)$, 相应地, $\forall p \in M, x_{0} \in X^{s}$, 方程 (1) 存在一解 $x_{p . x_{0}}-y_{p} s_{0}+x_{0}$, 满足

$$
\left\|x_{p, x_{0}}-x_{0}\right\| \leqslant \lambda^{-1}\left\|\tilde{D}_{p} \tilde{A}_{p}^{-1} \phi\left(x_{0}\right)\right\| \text {. }
$$

但由定理 1 知方程 (1) 仅有一解, 可见 $s:-x_{p, x_{0}}$ 与 $p, x_{0}$ 无关, 因而由(10)式可导出估计式 (4).

定理 3 如果(i) $X$ 是有限维空间; (ii) $A_{i}-A B_{i}, j-1,2, \cdots, m$, 这里 $A 、 B_{i} \in K^{\prime \times 1}$, 且 $A \neq 0$; (iii) 集合 $M_{B}-\left\{p \in \mathrm{N}: p \leqslant m, \operatorname{Det}\left(B_{p} D_{0}\right) \neq 0\right\}$ 非空; (iv) 存在 Hermite 矩阵 $R \in K^{\prime \times s}$ 及满足条件

$$
\Delta:-a_{2} b_{3}-a_{3} b_{2} \neq 0,0 \leqslant \operatorname{sgn}\left(a_{3}\right)+\operatorname{sgn}\left(b_{3}\right) \leqslant 1
$$

的实数 $a_{i}, b_{i}(i-1,2,3)$ 及 $e$, 使得

$$
Q_{0}>0, Q_{1} \geqslant 0, \sum_{i=1}^{3} a_{i} Q_{i} \geqslant 0, \sum_{i=1}^{3} b_{i} Q_{i} \geqslant 0,
$$

这里 $Q_{2}-\mathscr{D}^{*} \mathscr{B}+\mathscr{B} * \mathscr{D}-2 \mathscr{B}^{*}\left(R A+A^{*} R-e A^{*} R A\right) \mathscr{B}, Q_{3}-e\left(\mathscr{D} * \mathscr{B}+\mathscr{B}^{*} \mathscr{D}\right)$ $-29 B^{*} R \mathscr{B}, 9 B-\left[B_{1}, B_{2}, \cdots, B_{m}\right]$. 那么方程(1)有唯一解 $x \in X^{s}$, 且有估计 


$$
|| t-\sigma|| \leqslant \lambda^{-1} \min _{p \in \Psi_{B}}\left|\tilde{D}_{p} \widetilde{B}_{p}^{-1} \sum_{i=1}^{m} \tilde{B}_{i} \varphi_{i}(\sigma)\right| \mid \cdot
$$

证解的唯一性可由定理 1 直接推出,下证解的存在性及估计式(13). 令

$$
\left\{\begin{array}{c}
\tau_{k}-\frac{\left.\operatorname{sgn}\left(b_{3}-a_{3}\right) \Delta\right)}{k+|c|}, c_{k}-\frac{1}{1+e \tau_{k}}, \delta_{2}-\frac{c_{k}}{k+|e|}\left(\left|\frac{b_{3}}{\Delta}\right|-\frac{b_{k}}{\Delta(k+|e|)}\right), \\
\delta_{3}-\frac{c_{k}}{k+|c|}\left(\frac{a_{2}}{\Delta(k+|c|)}+\left|\frac{a_{3}}{\Delta}\right|\right), \\
\delta_{1}-1-a_{1} \delta_{2}-b_{1} \delta_{3}, \mathscr{\alpha}_{k}-\phi+\tau_{k} g_{B}, R_{k}-c_{k} R, k-1,2,3, \cdots .
\end{array}\right.
$$

从(11)、(12)、(14)式易知存在 $k_{0}>0$, 使当 $k>k_{0}$ 时有

$$
\delta_{i}>0, i-1,2,3 ; Q_{0}^{(k)}:-R_{k}-\sum_{i=1}^{m} \Lambda_{i}>0 ; \operatorname{Det}\left(A+\tau_{k} l\right) \neq 0,
$$

这里 $I$ 为 $s \times s$ 单位阵. 由此及(12)、(14)式进一步推出

$$
\begin{aligned}
Q_{i}^{(k)}:-\mathscr{D}^{*} \mathscr{C}_{k}+\mathscr{O}_{k}^{*} \mathscr{D}-2 \mathscr{C}_{k}^{*} R_{k} \mathscr{C}_{k}-Q_{1}+c_{k} \tau_{k} Q_{2}+c_{k} \tau_{k}^{2} Q_{3}-\delta_{1} Q_{1}+\delta_{2} \sum_{i=1}^{3} a_{i} Q_{i} \\
\quad+\delta_{3} \sum_{i=1}^{3} b_{i} Q_{i} \geqslant 0 .
\end{aligned}
$$

由于 $A_{i}-A B_{i}$, 且集合 $M_{B}$ 非空, 从(14)、(15)、(16)式及定理 2 推知, 当 $k>k_{0}$ 时, 方程

$$
x-\sigma-\sum_{i=1}^{m}\left(\tilde{A}+\tau_{k} \tilde{I}\right) \tilde{B}_{i} \varphi_{i}(x)=0
$$

有唯一解 $x(k) \in X^{\boldsymbol{t}}$, 满足

$$
\left\|x(k)-\sigma^{\|}\right\| \leqslant\left(\lambda_{m i D}^{Q_{0}^{(k)}}+\lambda_{\operatorname{miD}}^{Q_{1}^{(k)}} / \lambda_{\operatorname{mos}}^{2 \sigma_{k}^{*} k}\right)^{-1} \min _{p \in M_{B}} \| \widetilde{D}_{p} \widetilde{B}_{p}^{-1} \sum_{j=1}^{m} \widetilde{B}_{i}\left(\varphi_{i}(\sigma) \|_{0}\right.
$$

因 $X^{s}$ 是有限维空间, 从有界列 $\{x(k)\}$ 中可选出收敛于 $X^{s}$ 中某点 $s$ 的子列 $\left\{x\left(k_{*}\right)\right\}$. 在(17) 及(18)式中以 $k_{n}$ 代替 $k$, 以 $x\left(k_{n}\right)$ 代替 $x$, 然后令 $n \rightarrow \infty$, 由(14)式及诸 $\varphi_{i}$ 的连续性立得 欲证之结果.

当 $m=1$ 时, 记 $A_{1} 、 \varphi_{1} 、 D_{1} 、 A ＼mathrm{~ 为 ~} A 、 \varphi 、 D 、 \Lambda$, 方程 (1) 成为

$$
x-\sigma-\tilde{A} \varphi(x)-0 \text {. }
$$

推论 1 若存在 Hermite 矩阵 $R \in K^{s \times s}$, 使下列条件之一成立:

(i) $Q_{0}:-R-\Lambda>0, Q_{1}:-D^{*} A+A^{*} D-2 A^{*} R A \geqslant 0$;

(ii) $Q_{0} \geqslant 0, Q_{1}>0$;

(iii) $Q_{0} \geqslant 0, Q_{1} \geqslant 0$, 且 $\operatorname{Re}\langle\widetilde{D}(\varphi(y)-\varphi(z)), y-z\rangle\langle\langle y-z, X(y-z)\rangle, \forall y, z \epsilon$ $X^{\prime}, y \neq z$.

则(19)式至多有一个解:若条件(i)或(ii)成立, 且 $A, D$ 非奇,(19)式有唯一解 $x \in X^{s}$, 且满足

$\left\|\boldsymbol{z}-x_{0}\right\| \leqslant \lambda^{-1}\left\|\widetilde{D} \tilde{A}^{-1}\left(x_{0}-\sigma\right)-\widetilde{D}_{\varphi}\left(x_{0}\right)\right\|$ ( $\lambda$ 仍由(5)式确定,下同), $\forall x_{0} \in X^{s}$.

推论 2 设 $X$ 是有限维空间, $A \neq 0, D$ 非奇, 且存在非奇矩阵 $B \in K^{i \times s}$ 、Hermite 阵矩 $R \in K^{i \times s}$ 及满足条件 (11) 的实数 $a_{i}, b_{i}(i-1,2,3)$ 及 $e$, 使 (12)式成立, 但这里定义 $Q_{2}-$ $D^{*} B+B^{*} D-2\left(B^{*} R A+A^{*} R B-e A^{*} R A\right), Q_{3}-e\left(D^{*} B+B^{*} D\right)-2 B^{*} R B$. 则方程(19) 有唯一解 $\hat{x} \in X^{s}$, 且有估计: $\|\hat{x}-\sigma\| \leqslant 2^{-1}\left\|\tilde{D}_{\varphi}(\sigma)\right\|$.

在推论 2 中, 取 $a_{1}-e-l, a_{2}-b_{3}-1, a_{3}-b_{1}-b_{2}-0, R-l\left(B^{*-1} D^{*}+D B^{-1}\right) / 2$, 
得到

推论 3 设 $X$ 是有限维空间, $A \neq 0, D$ 非奇, 且存在非奇矩阵 $B \in K^{\prime \times d}$ 及实数 $l$, 使 $l\left(B^{*-1} D^{*}+D B^{-1}\right)-2 A>0,\left(I-l A^{*} B^{*-1}\right) D^{*} A+A^{*} D\left(I-l B^{-1} A\right) \geqslant 0$,

$$
\left(I-l A^{*} B^{*-1}\right) D^{*} B+B^{*} D\left(I-l B^{-1} A\right) \geqslant 0 \text {. }
$$

则方程(19)有唯一解 $\hat{x} \in X^{\prime}$, 且满足 $\|\hat{x}-\sigma\| \leqslant \lambda^{-1}\|\tilde{D} \varphi(\sigma)\|$.

取 $a_{1}=e-2 l, a_{2}-b_{3}-1, a_{3}-0, b_{1}=-2 l^{2}, b_{2}--l, R=l \hat{R}, B-\hat{R}^{-1} D$, 这 里 $R$ 是 Hermite 正定矩阵,由推论 2 得到

推论 4 设 $X$ 是有限维空间, $A \neq 0, D$ 非奇, 且存在 Hermite 正定矩阵 $R \in K^{\prime \times x}$ 及实 数 $l$,使

$$
l R-\Lambda>0, D^{*} A+A^{*} D-2 l A^{*} R A \geqslant 0 .
$$

则方程 (19)有唯一解 $\left\{\in X^{s}\right.$, 且满足 $\|\hat{A}-\sigma\| \leqslant \lambda^{-1}\|\widetilde{D} \varphi(\sigma)\|$.

以 $s$ 级 $r$ 值 $k$ 阶导数方法求解发展方程 $y^{\prime}(t)-f(y(t)), t \in \mathbf{R}$ 时, 每步需求解如下形 式的方程:

$$
Y^{(\star)}-\tilde{C}_{0} y^{(s)}+\sum_{i=1}^{m}(-1)^{q_{i}-1} h^{q_{i}} \widetilde{C}_{q i} \widetilde{G}_{q ;} F^{\left(q_{i}-1\right)}\left(Y^{(\star)}\right),
$$

这里设 $f: X \rightarrow X$ 充分光滑, $\left\{q_{1}, q_{2}, \cdots, q_{m}\right\} \subset\{1,2, \cdots, k\}, C_{0}, C_{q i}, G_{a i}$ 是系数矩阵, 其中 $G_{i j}$ 是非负对角阵, $y^{(n)} \in X^{r}, Y^{(n)} \in X^{r}$,

$$
\left\{\begin{array}{c}
F^{(q)}(Y)-\left(f^{(q)}\left(Y_{1}\right), f^{(q)}\left(Y_{2}\right), \cdots, f^{(q)}\left(Y_{s}\right)\right) \in X^{s}, \forall Y-\left(Y_{1}, Y_{2}, \cdots, Y_{s}\right) \in X^{s} ; \\
f^{(0)}(y)-f(y), f^{(q)}(y)-\left(\frac{\mathrm{d}}{\mathrm{d} y} f^{(q-1)}(y)\right) f(y), q-1,2,3, \cdots, y \in X ; \\
(-1)^{q-1} \operatorname{Re}\left\langle f^{(q-1)}(y)-f^{(q-1)}(z), y-z\right\rangle \leqslant \mu_{q}\|y-z\|^{2}, \forall y, z \in X, \\
q-q_{1}, q_{2}, \cdots, q_{m .} .
\end{array}\right.
$$

方程(20)可写成(1)式的形式,只要令

$$
x-Y^{(n)}, \sigma-\widetilde{C}_{0} y^{(n)}, A_{i}-C_{q i}, \varphi_{i}(x)=(-1)^{q_{i}^{-1}} h^{q} ; \widetilde{G}_{q i} F^{\left(q_{i}-1\right)}(x) .
$$

由于(21)式成立, 任何 $s \times s$ 对角矩阵 $D_{i} \geqslant 0$ 及 $\Lambda_{i}-h^{q_{i}} \mu_{q_{i}} D_{i} G_{q i}$ 均可作为 $\varphi_{i}$ 的示性矩 阵. 因此, 本文结果直接适用于多值多导法; 特别适用于 Runge-Kutta 方程. 不难看出文献 $[2,3]$ 中结果及文献 [4]中主要结果均可视为本文推论 $1 、 4$ 之特例.

\section{参考文塥}

[1] Dolph, C. \& Minty, G., Nonlinear Integral Eqwations (Ed. Anselone, P.), Univ. of Wisconsin Press, Madison, iVifconsin, 1964, 99-154.

[ 2] Coper, G. J., IMA J. Numer. Anal., 6(1986), 325-330.

[3] Kuang Jiaoxun \& Xiang Jiaxiang. BIT, 29(1989), 321-327.

(4) Crouzeix. M., Hundsdorfer, W. H. \& Spijker, M N., BIT, 23(1983), 84-91. 